\title{
Evolution of Segregation Distortion: Potential for a High Degree of Polymorphism
}

\author{
Michiel van Boven and Franz J. Weissing* \\ Department of Genetics, University of Groningen, P.O. Box 14, 9750 AA Haren, \\ The Netherlands
}

(Received on 3 February 1997, Accepted in revised form on 2 July 1997)

\begin{abstract}
By means of a population genetical model, we study the evolution of segregation distortion. Most models of segregation distortion focus on a single distorter allele. In contrast, we consider the competition between a large number of distorters. Motivated by systems as the $t$ complex of the house mouse or the $S d$ complex of Drosophila melanogaster, we assume that there is some "complementation" between distorter alleles, i.e. that the fitness of individuals heterozygous for two distorter alleles is higher than the fitness of homozygous individuals. In the presence of complementation, the most efficient distorter allele with the highest segregation ratio often does not outcompete less efficient distorters. In fact, our results show that coexistence of a large number of distorter alleles is more typical than the competitive exclusion of less efficient distorters by a single superior allele. We first consider the analytically tractable system where all distorters show the same amount of complementation. In this case, all distorters with a segregation ratio higher than a certain critical value will persist, resulting in a polymorphic population where the average segregation ratio is only slightly larger than 0.5 . If the degree of complementation varies, there may be more than one stable equilibrium, and the outcome of competition may depend on the initial conditions. Motivated by empirical examples, we also consider the case that the distorting ability of an allele is negatively related to its effects on individual fitness. Interestingly, the outcome of competition depends crucially on details of such a trade-off. We conclude that verbal arguments are insufficient to predict the evolution of segregation distortion.
\end{abstract}

(C) 1998 Academic Press Limited

\section{Introduction}

The evolution of segregation distortion is governed by selection at different levels. Their segregation advantage notwithstanding, all known segregation distorters do not spread to fixation since they induce severe negative fitness effects at the individual level. If gamete and individual level are opposed to one another, the interaction between the wildtype allele and a distorter allele will often result in a stable polymorphism. This intuitive prediction is confirmed by a large number of models, both for an infinite population (e.g., Bruck, 1957; Lewontin \& Dunn, 1960; Petras, 1967; Lewontin, 1968; Hartl, 1970a;

\footnotetext{
*Author to whom correspondence should be addressed. E-mail: weissing@biol.rug.nl
}

Feldman \& Otto, 1991), and for a structured metapopulation (e.g., Lewontin \& Dunn, 1960; Lewontin, 1962; Nunney \& Baker, 1993; van Boven \& Weissing, 1998b).

All these models focus on the dynamics of a single distorter allele. Therefore, they are not suited to address questions concerning the long-term evolution of segregation distortion. It has been conjectured, on the basis of verbal arguments, that in the course of evolution more efficient distorters with a high segregation ratio will replace those with a lower segregation ratio (e.g., Silver, 1985, 1993). Whether this hypothesis is reasonable, however, needs to be tested on the basis of models which explicitly take the competition between different distorter alleles into account. 
Recently, van Boven et al. (1996) and Weissing \& van Boven (1998) showed that the outcome of competition is often not obvious in the case of two distorter alleles. In particular, a relatively inefficient distorter allele with a low segregation ratio can easily coexist with a highly efficient distorter allele with a high segregation ratio. The main prerequisite for coexistence is the well-documented phenomenon of "complementation", i.e. the fact that the fitness of individuals heterozygous for two different distorter alleles is higher than the fitness of individuals homozygous for a distorter allele (e.g., Temin et al., 1991; Lyon, 1991).

Here we extend the analysis of van Boven et al. (1996) and Weissing \& van Boven (1998) by considering the competition between a large number of distorter alleles (see also Hartl, 1970b; Liberman, 1991; Godelle \& Reboud, 1997). We show that stable coexistence is not restricted to a small number of distorter alleles. On the contrary, in the presence of complementation an unlimited number of alleles may coexist, resulting in a highly polymorphic population.

The structure of this paper is as follows. We present a model for selection and segregation distortion in an infinitely large randomly mating population with one sex. To fix ideas, we first consider the competition between the wildtype and two distorter alleles. We then show how the analysis can be extended to the competition between a large number of distorters. We start by assuming that the amount of complementation is the same for all combinations of distorter alleles. In this case, each parameter configuration results in a unique stable polymorphism. We give an analytical characterization of this equilibrium, and show that it typically involves many alleles. Subsequently, we show by means of a simple example that the outcome of competition may be contingent on the initial conditions if the degree of complementation differs between distorters. Finally, we study the competition between segregation distorters in case that there is a negative trade-off between distorting efficiency and complementing ability.

The analysis will reveal that the outcome of evolution is strongly affected by the amount and pattern of complementation. If there is no complementation at all, the most efficient distorter outcompetes all less efficient alleles, while if there is strong complementation a high degree of polymorphism is expected. In the latter case, the evolutionary success of a segregation distorter may be determined more by its ability to complement other alleles than by its segregation ratio.

\section{The Model}

We consider selection and segregation distortion at an autosomal locus in an infinitely large, randomly mating population with one sex only. Generations are discrete and non-overlapping. Let us define the fitness of allele $A_{i}$ in an $A_{i} A_{j}$ individual by the product of the viability $v_{i j}$, the (multiplicative) fertility $f_{i j}$, and the segregation ratio $s_{i j}$ of $A_{i}$ in an $A_{i} A_{j}$ individual:

$$
w_{i j}=v_{i j} f_{i j} s_{i j} .
$$

If $p_{i}$ denotes the relative frequency of allele $A_{i}$ in the gametes (after segregation distortion has taken place), the change in allele frequency from one generation to the next is given by [see e.g., Liberman (1991) or Weissing \& van Boven (1998)]:

$$
p_{i}^{\prime}=p_{i} \frac{w_{i}(\mathbf{p})}{\bar{w}(\mathbf{p})} .
$$

Here

$$
w_{i}(\mathbf{p})=\sum_{l} w_{i l} p_{l}
$$

and

$$
\bar{w}(\mathbf{p})=\sum_{k, l} p_{k} w_{k} p_{l}
$$

represent the marginal fitness of allele $A_{i}$ and the mean fitness of the population respectively.

We make the standard assumption that the viability and fertility parameters are symmetric (i.e. $v_{i j}=v_{j i}$ and $f_{i j}=f_{j i}$, while the segregation parameters satisfy $s_{i j}+s_{j i}=1$. In the absence of segregation distortion (i.e. $s_{i j}=s_{j i}=\frac{1}{2}$ for all $\left.i, j\right)$, the fitness matrix $\mathbf{W}=\left(w_{i j}\right)$ is symmetric and (2) corresponds to the classical Haldane-Fisher-Wright model for selection at an autosomal locus (e.g., Nagylaki, 1992). In the presence of segregation distortion, however, $\mathbf{W}$ is asymmetric and $w_{i j}$ should be interpreted as the fitness of an $A_{i} A_{j}$ individual viewed from the perspective of $A_{i}$. In this case, (2) corresponds to the discrete replicator dynamics (e.g., Hofbauer \& Sigmund, 1988; Weissing, 1991), as was first pointed out by Liberman (1991).

In the following, we focus on a single wildtype allele $A_{0}$ and a number of distorter alleles $A_{1}, \ldots, A_{n}$. Motivated by empirical examples such as the $t$ complex of the house mouse (e.g., Silver, 1993) or the $S d$ complex of Drosophila melanogaster (e.g., Temin et al., 1991), we assume throughout that segregation is only distorted in heterozygous $A_{i} A_{0}$ individuals: the segregation ratio of $A_{i}(i \geq 1)$ in combination with the wildtype $\left(s_{i 0}\right)$ will be denoted by $\sigma_{i}$. Furthermore, we assume that the viability and fertility of individuals 
carrying at least one wildtype allele is not impaired (i.e. $v_{i 0}=f_{i 0}=1$ ). As a result, $w_{00}=\frac{1}{2}, w_{i 0}=\sigma_{i}$, and $w_{0 i}=1-\sigma_{i}$. The fitness of individuals homozygous for a distorter allele is assumed to be zero: $w_{i i}=0$ $(i \geq 1)$. Finally, the parameters $w_{i j}$ with $i, j \geq 1$ and $i \neq j$ relate to the fitness of an allele in individuals heterozygous for two different distorters: $w_{i j}=\frac{1}{2} v_{i j} f_{i j}=w_{j i}$. We say that $A_{i}$ and $A_{j}$ 'complement' another if the fitness of $A_{i} A_{j}$ heterozygotes is higher than that of $A_{i} A_{i}$ or $A_{j} A_{j}$ homozygotes. If there is no complementation, then $w_{i j}=0$. If there is full complementation, then $w_{i j}=\frac{1}{2}$.

\section{Competition Between Two Distorters}

Let us start by considering the competition between two segregation distorters [see van Boven et al. (1996) and Weissing \& van Boven (1998) for a more general analysis]. We will focus on the interaction of a wildtype allele $A_{0}$ and two distorter alleles $A_{1}$ and $A_{2}$. In this case, the fitness matrix $\mathbf{W}=\left(w_{i j}\right)$ is given by

$$
\mathbf{W}=\left(\begin{array}{ccc}
\frac{1}{2} & 1-\sigma_{1} & 1-\sigma_{2} \\
\sigma_{1} & 0 & \alpha \\
\sigma_{2} & \alpha & 0
\end{array}\right]
$$

Here $\alpha=w_{12}\left(0 \leq \alpha \leq \frac{1}{2}\right)$ is the amount of complementation between the distorter alleles $A_{1}$ and $A_{2}$.

At an equilibrium of (2), an allele $A_{i}$ with $p_{i}^{*}>0$ satisfies $w_{i}\left(\mathbf{p}^{*}\right)=\bar{w}\left(\mathbf{p}^{*}\right)$. Hence, at equilibrium either the frequency of an allele is zero, or its marginal fitness equals the mean fitness of the population. For the fitness matrix (3), the three border equilibria $\left(p_{0}^{*}, p_{1}^{*}, p_{2}^{*}\right)^{T}$ with $p_{0}^{*}=0, p_{1}^{*}=0$, or $p_{2}^{*}=0$ are given by $\left(0, \frac{1}{2}, \frac{1}{2}\right)^{T},\left(2-2 \sigma_{2}, 0,2 \sigma_{2}-1\right)^{T}$, and $\left(2-2 \sigma_{1} 2 \sigma_{1}-1\right.$, $0)^{T}$, respectively. A straightforward calculation shows that the fully polymorphic equilibrium $\mathbf{p}^{*}=\left(p_{0}^{*}, p_{1}^{*}, p_{2}^{*}\right)^{T}$ is given by

$$
\begin{array}{r}
p_{0}^{*}=\frac{\alpha(1-\alpha)+\alpha\left(1-\sigma_{1}-\sigma_{2}\right)}{\alpha(1-\alpha)+\left(\sigma_{1}-\sigma_{2}\right)^{2}} \\
p_{1}^{*}=\frac{\alpha\left(\sigma_{2}-\frac{1}{2}\right)+\left(1-\sigma_{2}\right)\left(\sigma_{1}-\sigma_{2}\right)}{\alpha(1-\alpha)+\left(\sigma_{1}-\sigma_{2}\right)^{2}} \\
p_{2}^{*}=\frac{\alpha\left(\sigma_{1}-\frac{1}{2}\right)+\left(1-\sigma_{1}\right)\left(\sigma_{2}-\sigma_{1}\right)}{\alpha(1-\alpha)+\left(\sigma_{1}-\sigma_{2}\right)^{2}} .
\end{array}
$$

Since the denominators are positive, this interior equilibrium exists whenever

$$
\begin{gathered}
\alpha<2-\sigma_{1}-\sigma_{2}, \quad \sigma_{1}>\sigma_{2}-\frac{\sigma_{2}-\frac{1}{2}}{1-\sigma_{2}} \alpha, \\
\sigma_{2}>\sigma_{1}-\frac{\sigma_{1}-\frac{1}{2}}{1-\sigma_{1}} \alpha .
\end{gathered}
$$

It is easy to show that these conditions for the existence of a polymorphic equilibrium are actually equivalent to the conditions for instability of the border equilibria with respect to an invasion attempt of the allele not present at equilibrium. In other words, the external instability of all border equilibria guarantees the existence of a fully polymorphic equilibrium with $p_{i}^{*}>0$ for $i \in\{0,1,2\}$. Moreover, it can be shown that this polymorphic equilibrium, if it exists, is always stable (Weissing \& van Boven, unpublished).

Using the relations (5), we can now classify for which parameter combinations a fully polymorphic equilibrium exists (in which case it is the only stable equilibrium). In the same manner, we can determine for which parameter combinations the equilibria involving only one allele or two alleles are stable. The results are given in Fig. 1. For a given amount of complementation $(\alpha=0.05)$, this figure shows which alleles will coexist as a function of the segregation parameters $\sigma_{1}$ and $\sigma_{2}$. If both distorters have a segregation disadvantage $\left(\sigma_{1}<\frac{1}{2}\right.$ and $\left.\sigma_{2}<\frac{1}{2}\right)$, only the wildtype will persist. If both distorters have a very high segregation ratio, they may coexist without the wildtype. If one distorter is moderately efficient (e.g., $\left.\sigma_{1}=0.75\right)$ and the other is relatively inefficient (e.g., $\sigma_{2}=0.60$ ), only the more efficient distorter will coexist with the wildtype. Coexistence of all three

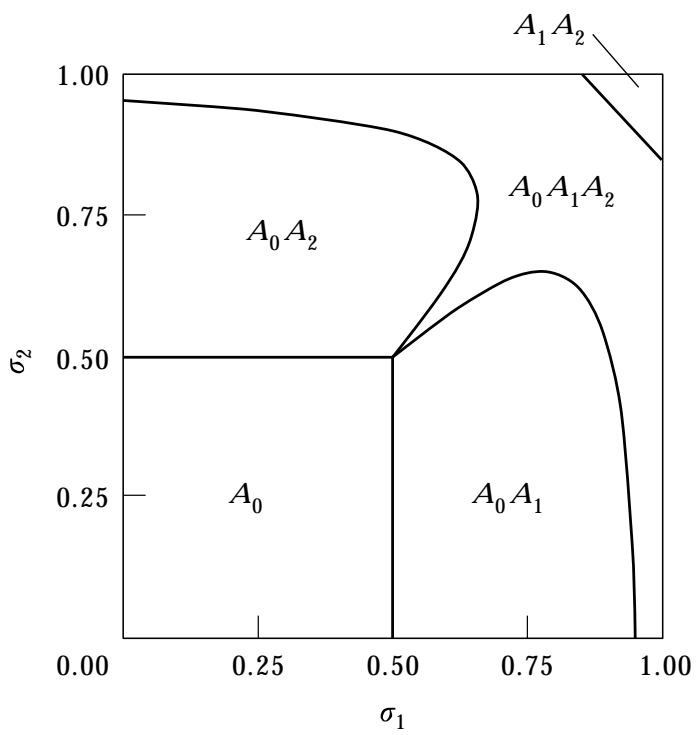

FIG. 1. Outcome of competition between a wildtype allele $A_{0}$ and two segregation distorters $A_{1}$ and $A_{2}$ as a function of the segregation ratios $\sigma_{1}$ and $\sigma_{2}$. The fitness matrix $\mathbf{W}$ is given by eqn (3) with $\alpha=0.05$. Five equilibrium outcomes are possible: only the wildtype persists $\left(A_{0}\right)$, the wildtype reaches a stable equilibrium with one distorter allele $\left(A_{0} A_{1}\right.$ and $\left.A_{0} A_{2}\right)$, the wildtype reaches a stable equilibrium with both distorters $\left(A_{0} A_{1} A_{2}\right)$, or both distorters reach a stable equilibrium without the wildtype $\left(A_{1} A_{2}\right)$. 
alleles may occur if the segregation ratios of the two distorters are similar $\left(\sigma_{1} \approx \sigma_{2}\right)$, or if one distorter has a particularly high segregation ratio. The latter case may seem surprising, but can be understood as follows. Imagine a very efficient distorter $A_{1}$ with a high segregation ratio that is present in a wildtype population. Such a distorter will reach a high frequency which is related to its segregation ratio $\left(p_{1}^{*}=2 \sigma_{1}-1\right)$. Consider now an invasion attempt by a second distorter allele. Since the efficient distorter is prevalent in the population, the fate of the second distorter is mainly determined by its interaction with the first distorter allele. As soon as there is some complementation between the two distorter alleles, the second distorter may hike along with the first distorter, even if it has no segregation advantage. In other words, the fate of a second distorter may be determined more by its ability to complement the efficient distorter than by its efficiency to exploit the wildtype (which is present in low frequency). If the segregation ratio of the first distorter is very high (here $\sigma_{1}>0.9756$ ), the segregation ratio of the second distorter even becomes irrelevant for its ability to persist stably.

\section{Competition Between Many Distorters: Equal Complementation}

Let us now consider the competition between a large number of segregation distorters. We assume that there is a fixed amount of complementation between all distorter alleles, and focus on the evolution of the segregation ratio. More precisely, we focus on the competition between a wildtype $A_{0}$ and $n$ distorter alleles $A_{1}, \ldots, A_{n}$, where the fitness matrix $\mathbf{W}$ is given by

$$
\mathbf{W}=\left(\begin{array}{ccccc}
\frac{1}{2} & 1-\sigma_{1} & 1-\sigma_{2} & \ldots & 1-\sigma_{n} \\
\sigma_{1} & 0 & \alpha & \ldots & \alpha \\
\sigma_{2} & \alpha & 0 & \ddots & \vdots \\
\vdots & \vdots & \ddots & \ddots & \alpha \\
\sigma_{n} & \alpha & \ldots & \alpha & 0
\end{array}\right)
$$

Here, the fitness of allele $A_{i}$ or allele $A_{j}$ in a compound $A_{i} A_{j}$ individual is denoted by $w_{i j}=\alpha$ for all $i \neq j$ $(i, j \geq 1)$.

Figure 2 shows the outcome of competition between 11 distorter alleles. The segregation ratios of the distorters are spread evenly in the interval $[0,1]$. In (a) there is no complementation $(\alpha=0)$, in (b) there is some complementation $(\alpha=0.125)$, and in (c) and (d) there are even higher degrees of complementation $[\alpha=0.25$ in (c), and $\alpha=0.375$ in (d)]. At $t=0$, all alleles are present in equal frequencies. The figure shows that complementation is a potent force enhancing the coexistence of segregation distorters. Without complementation (a), the most efficient distorter (i.e. $\sigma=1.0$ ) outcompetes all other alleles, while if there is strong complementation (d), all distorter alleles (even those with $\sigma_{i}<\frac{1}{2}$ ) will persist stably. Still, the most efficient distorters with the highest segregation ratios reach the highest frequencies. This is validated in the Appendix by showing that, at equilibrium, differences in allele frequencies are related to the segregation ratios in the following manner:

$$
p_{i}^{*}-p_{j}^{*}=\left(\sigma_{i}-\sigma_{j}\right) \frac{p_{0}^{*}}{\alpha} .
$$

Hence, the differences in allele frequences are proportional to the differences in respective segregation ratios. Moreover, these differences are positively related to the equilibrium frequency of the wildtype, and inversely related to the amount of complementation. We may conclude that if the amount of complementation is equal for all distorters, the segregation ratio is an indicator of the relative success (i.e. the frequency) of a segregation distorter.

However, the segregation ratio per se does not yet predict whether a distorter can or cannot persist. As shown in the Appendix, a rare distorter allele $A_{n+1}$ will successfully invade a population in which $n$ distorter alleles are already present if and only if

$$
\sigma_{n+1}>\bar{\sigma}-\frac{\frac{\alpha}{n}\left(\bar{\sigma}-\frac{1}{2}\right)+\operatorname{Var}(\sigma)}{1-\bar{\sigma}-\alpha\left(1-\frac{1}{n}\right)} .
$$

Here

$$
\bar{\sigma}=\frac{1}{n} \sum_{k=1}^{n} \sigma_{k}
$$

and

$$
\operatorname{Var}(\sigma)=\frac{1}{n} \sum_{k=1}^{n}\left(\sigma_{k}-\bar{\sigma}\right)^{2}
$$

represent the unweighted mean and variance of the segregation ratios in the population. In view of (8), a rare distorter allele may invade even if its segregation ratio is smaller than that of the resident distorters. Furthermore, (8) indicates that the invasion prospects of a rare distorter are enhanced by a high degree of complementation, and by a large variance in the segregation ratios of the resident alleles.

In case that a large number of distorters is already present in the population, (8) can be used to determine the minimal segregation ratio that a rare 
(a)

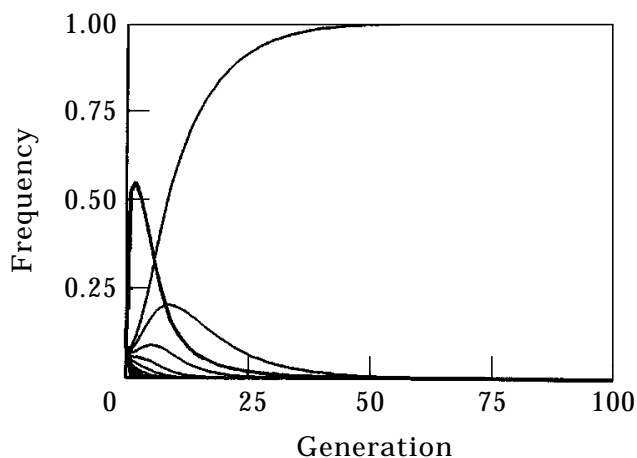

(c)

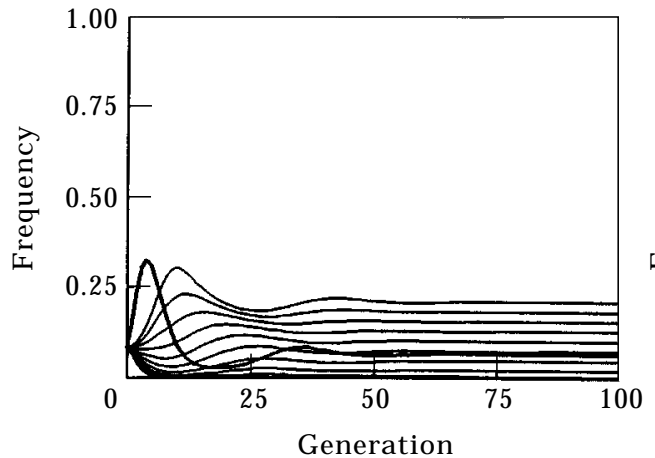

(b)

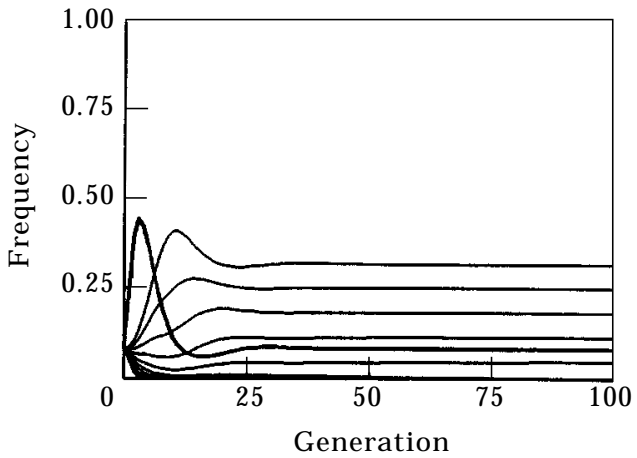

(d)

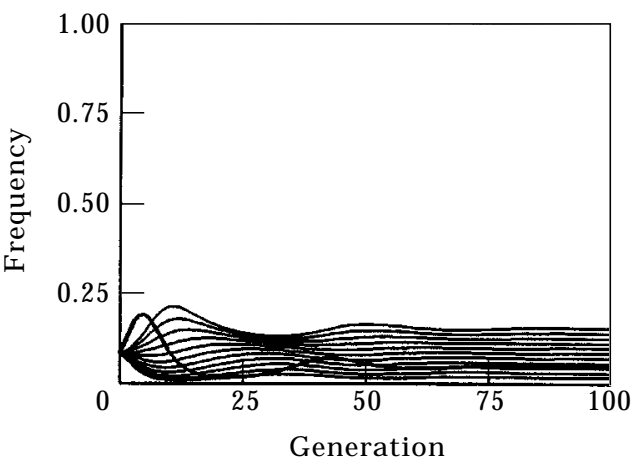

FIG. 2. Competition between the wildtype allele $A_{0}$ (bold line), and a number of distorter alleles $A_{i}(i=1, \ldots, 11)$. The segregation ratios of the distorter alleles are given by $\sigma_{i}=(i-1) / 10$ (i.e. the segregation ratio is increased from 0 to 1 with stepsize 0.1$)$. In (a) there is no complementation (i.e. $w_{i j}=0$ for $i, j \geq 1$ ), while there is an increasing amount of complementation in the other panels: $w_{i j}=0.125$ in (b), $w_{i j}=0.25$ in (c), and $w_{i j}=0.375$ in (d). In homozygous condition all distorters have zero fitness: $w_{i i}=0$. At $t=0$ all alleles are present in equal frequencies.

distorter allele needs for successful invasion. As shown in the Appendix, this minimal segregation ratio $\sigma_{\min }$ is given by (see Fig. 3)

$$
\sigma_{\min }(\alpha)=1-3 \alpha .
$$

This equation shows again that complementation facilitates the persistence of segregation distorters. Notice that for $\alpha>\frac{1}{6}$, distorters can be found that have a segregation disadvantage $\left(\sigma<\frac{1}{2}\right)$ but are still able to invade. Moreover, for $\alpha>\frac{1}{3}$, all distorters will successfully invade, regardless of their segregation ratio.

At equilibrium, the (weighted) mean segregation ratio in the population is given by

$$
\overline{p^{*} \sigma}=\sum_{i=1}^{n} p_{i}^{*} \sigma_{i}
$$

In a highly polymorphic population there is a simple relation between the degree of complementation and the mean segregation ratio (Appendix; Fig. 3):

$$
\overline{p^{*} \sigma}=1-\alpha .
$$

Accordingly, the (weighted) mean segregation ratio approaches the Mendelian value $\frac{1}{2}$ if there is full complementation $\left(\alpha=\frac{1}{2}\right)$. But even if $\alpha$ is relatively small, this need not imply that segregation distortion occurs frequently. In fact, since the frequency of the wildtype is usually quite low [see eqn (A.6)], most individuals will be heterozygous for two distorter alleles, and in these individuals segregation is Mendelian.

In principle, eqns (9) and (10) only apply if the number of distorters is infinitely large. However, simulations show that (9) and (10) already accurately predict $\sigma_{\min }$ and $\overline{p^{*} \sigma}$ if the number of resident distorter alleles is rather small ( $n \geq 5$, data not shown).

\section{Competition Between Three Distorters: Unequal Complementation}

Let us now illustrate, by means of a simple example, how the results may be affected if the 
amount of complementation is not equal for all distorter alleles. To this end, we consider the competition between a wildtype and three distorter alleles. In this example, the fitness matrix $\mathbf{W}$ is given by

$$
\mathbf{W}=\left(\begin{array}{cccc}
\frac{1}{2} & 1-\sigma_{1} & 1-\sigma_{2} & 1-\sigma_{3} \\
\sigma_{1} & 0 & \alpha & 0 \\
\sigma_{2} & \alpha & 0 & 0 \\
\sigma_{3} & 0 & 0 & 0
\end{array}\right)
$$

Here the distorter alleles $A_{1}$ and $A_{2}$ complement another to some degree $\alpha$, while the third distorter $A_{3}$ does not show any complementation in combination with $A_{1}$ or $A_{2}$.

Consider first an equilibrium population of the wildtype $A_{0}$ and the third distorter allele $A_{3}$ $\left(p_{3}^{*}=1-p_{0}^{*}=2 \sigma_{3}-1\right)$. Such a population will be destabilized by an invasion attempt of $A_{1}$ whenever the marginal fitness of $A_{1}$ exceeds the marginal fitness of $A_{3}$. Since $w_{1}\left(\mathbf{p}^{*}\right)=\sigma_{1} p_{0}^{*}$ and $w_{3}\left(\mathbf{p}^{*}\right)=\sigma_{3} p_{0}^{*}$ [see eqn (A.2) with $\alpha=0$ ], it is clear that a rare distorter $A_{1}$ will increase in frequency if and only if it is superior with respect to its segregation ratio: $\sigma_{1}>\sigma_{3}$. Similarly, a rare allele $A_{2}$ will increase if and only if $\sigma_{2}>\sigma_{3}$. Hence the resident population can be destabilized by $A_{1}$ or $A_{2}$ if and only if

$$
\sigma_{3}<\max \left\{\sigma_{1}, \sigma_{2}\right\} .
$$

On the other hand, an equilibrium population consisting of the wildtype and two complementing distorters $A_{1}$ and $A_{2}$ can be invaded successfully by $A_{3}$

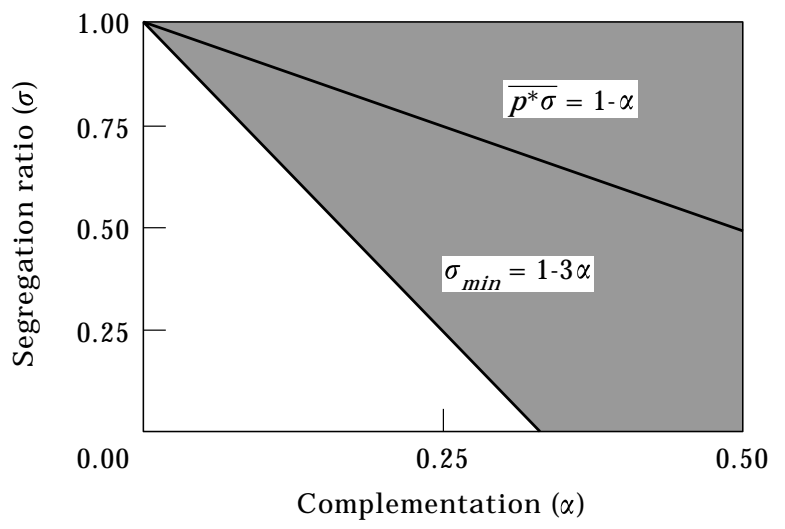

FIG. 3. Outcome of competition between a large number of distorter alleles as a function of the amount of complementation. All distorter alleles complement another to the same degree: $w_{i j}=\alpha$ $(i, j \geq 1, i \neq j)$. The hatched area represents the range of distorters that are able to invade the population when a large number of distorters is already present. The minimal segregation ratio $\sigma_{\min }(\alpha)$ needed for successful invasion is given by eqn (9). whenever, at equilibrium, the marginal fitness of $A_{3}$ is larger than that of any of the resident alleles $\left(w_{3}\left(\mathbf{p}^{*}\right)>w_{i}\left(\mathbf{p}^{*}\right)\right.$ for $i \in\{0,1,2\}$; see (A.2)). Since $w_{3}\left(\mathbf{p}^{*}\right)=\sigma_{3} p_{0}^{*}$, a simple calculation shows that $A_{3}$ will invade whenever

$$
\sigma_{3}>\bar{\sigma}+\frac{\alpha}{2} \frac{1-p_{0}^{*}}{p_{0}^{*}}
$$

where $\bar{\sigma}=\frac{1}{2}\left(\sigma_{1}+\sigma_{2}\right)$.

It is conceivable that $A_{3}$, although superior with respect to its distortion ability, is nevertheless not able to invade a population where $A_{1}$ and $A_{2}$ are already present. This may happen whenever

$$
\max \left\{\sigma_{1}, \sigma_{2}\right\}<\sigma_{3}<\frac{1}{2}\left(\sigma_{1}+\sigma_{2}\right)+\frac{\alpha}{2} \frac{1-p_{0}^{*}}{p_{0}^{*}} .
$$

In such a situation (which is favoured by a high degree of complementation between $A_{1}$ and $A_{2}$, and a low wildtype frequency) there are two stable equilibria, one involving the wildtype and the efficient distorter $A_{3}$, and one involving the wildtype and $A_{1}$ and $A_{2}$. Consequently, the outcome of competition may depend on the initial conditions in these cases. This is illustrated by Fig. 4 where at $t=0$ three distorters are introduced with equal frequency into a wildtype population. The first and second distorters $A_{1}$ and $A_{2}$ do not have a high segregation ratio $\left(\sigma_{1}=0.70, \sigma_{2}=0.75\right)$ but complement another fully $\left(w_{12}=\frac{1}{2}\right)$, while the third distorter $A_{3}$ has a high segregation ratio $\left(\sigma_{3}=0.95\right)$, but does not complement the other alleles $\left(w_{13}=w_{23}=0\right)$. In (a) the initial frequency of all distorters is 0.01 , while it is 0.02 in (b). Initially all three distorters increase in frequency at the expense of the wildtype. The most efficient distorter with the highest segregation ratio, however, increases more rapidly than the two less efficient ones. In Fig. 4(a) this leads to the competitive exclusion of the two distorters with a lower segregation ratio. In Fig. 4(b), on the other hand, the initial frequency of all distorters is somewhat higher, and this allows the two complementing distorters to increase further in frequency after the initial phase. Here, the more efficient distorter is outcompeted by the joint action of the less efficient ones.

\section{Trade-offs Between Distortion Efficiency and Individual Fitness}

Up to now, we considered complementation as a factor that is independent of segregation distortion. This assumption may not be realistic. For the $t$ complex of the house mouse and the $S d$ complex of Drosophila melanogaster, empirical evidence suggests that the negative fitness effects at the individual level 


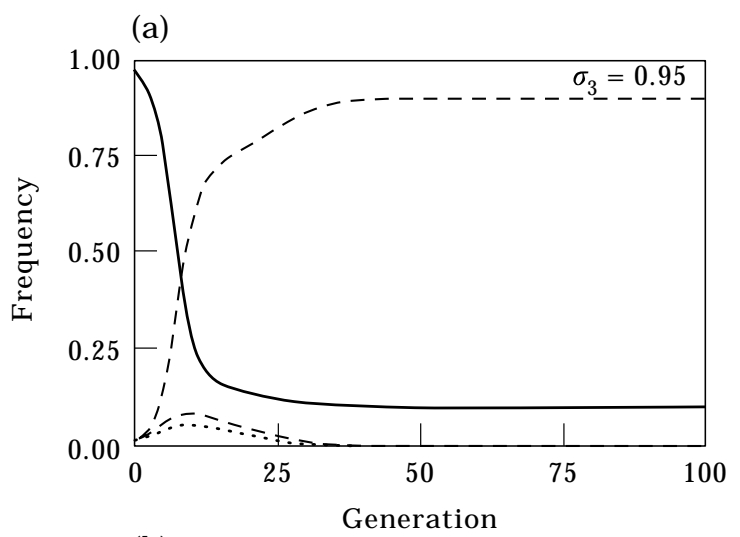

(b)

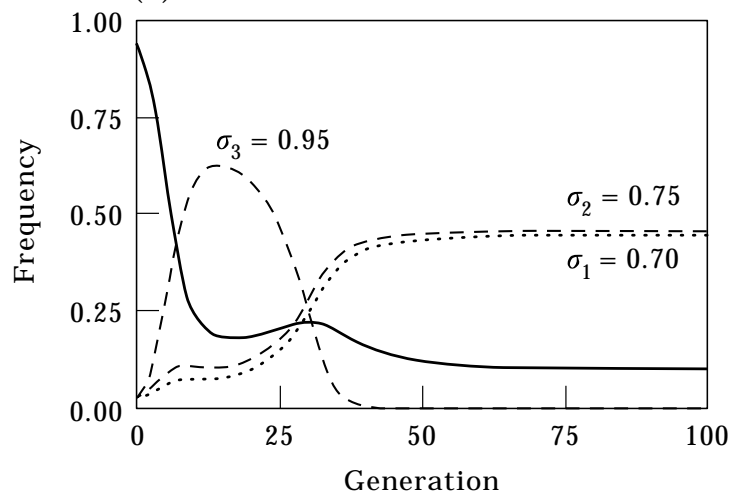

FIG. 4. Dependence of the outcome of competition on the initial conditions. Two distorters of intermediate efficiency $\left(\sigma_{1}=0.70\right.$, $\left.\sigma_{2}=0.75\right)$ that complement another well $\left(w_{12}=0.50\right)$ and an efficient third distorter $\left(\sigma_{3}=0.95\right)$ that does not show any complementation $\left(w_{13}=w_{23}=0\right)$ are introduced simultaneously into the population with low frequency. The initial frequency of all distorters is 0.01 in (a), and 0.02 in (b).

(mainly male sterility) are a direct consequence of the phenomenon of segregation distortion (Temin et al., 1991; Lyon, 1991). In both systems, a high distorting ability seems to induce more severe negative fitness consequences at the individual level. Loosely speaking, the distorter alleles manage to disable competing gametes (typically the spermatozoa). Individuals homozygous for a distorter or heterozygous for two distorters produce gametes that harm each other, hence leading to a reduced fitness (usually male fertility).

The details of the trade-off between segregation distortion and individual fitness (i.e. viability times fertility) are not yet well understood [but see Hurst (1993) for a speculative mechanistic model]. Therefore, we will here consider two simple scenarios. We assume that the individual fitness $2 w_{i j}$ (viability times fertility) of an $A_{i} A_{j}$ individual $(i, j \geq 1, i \neq j)$ is negatively related to the segregation ratios $\sigma_{\mathrm{i}}$ and $\sigma_{\mathrm{j}}$. More specifically, we consider the two models

$$
2 w_{i j}=\left(1-\sigma_{i}^{k}\right)\left(1-\sigma_{j}^{k}\right)
$$

and

$$
2 w_{i j}=1-\sigma_{i}^{k} \sigma_{j}^{k},
$$

that will be called the "multiplicative fitness model" and the "epistatic fitness model", respectively. In both models, a distorter with $\sigma=0.0$ does not depress fitness at all, while a distorter with $\sigma=1.0$ depresses fitness maximally. It is obvious that smaller values of the parameter $k$ lead to more severe negative fitness effects than larger values of $k$. The main difference between the two models is that for two given segregation ratios the amount of complementation is always higher in the epistatic fitness model (16) than in the multiplicative fitness model (15).

Let us start by considering the competition between two distorter alleles. As indicated above, the two distorters will stably coexist whenever they are able to spread when rare. The invasion criterion (5) [or (8) with $n=1$ ] shows that this is the case if

$$
\sigma_{2}>\sigma_{1}-\frac{\sigma_{1}-\frac{1}{2}}{1-\sigma_{1}} w_{12} \text { and } \sigma_{1}>\sigma_{2}-\frac{\sigma_{2}-\frac{1}{2}}{1-\sigma_{2}} w_{12},
$$

or equivalently,

$$
\frac{\sigma_{1}-\frac{1}{2}}{1-\sigma_{1}}>\frac{\sigma_{1}-\sigma_{2}}{w_{12}}>\frac{\sigma_{2}-\frac{1}{2}}{1-\sigma_{2}} .
$$

Insertion of eqns (15) or (16) into eqn (17) yields the parameter combinations for which stable coexistence of all three alleles is possible. Figure 5 shows the results for the multiplicative [(a) and (b)] and for the epistatic [(c) and (d)] model in case of severe $\left[k=\frac{1}{2}\right.$, (a) and (c)] and less severe $[k=2$, (c) and (d)] fitness reduction. Notice that coexistence of the distorter alleles depends strongly on the details of the trade-off between distortion efficiency and individual fitness. In the multiplicative model with its stronger negative fitness consequences, only positive distorters (i.e. $\sigma_{\mathrm{i}}>\frac{1}{2}$ ) can stably persist, and stable coexistence of two distorter alleles is only possible if the two segregation ratios do not differ much. In contrast, stably coexistence is easily achieved in the epistatic model: Here a negative distorter may well persist stably with a highly efficient distorter. In extreme cases it is even possible that a negative distorter (e.g., $\sigma=0.10$ ) may profit from decreasing its segregation ratio even more (e.g., to $\sigma=0.0$ ) to increase the amount of complementation in combination with an efficient distorter [Fig. 5(c)].

Hence, in case of two distorter alleles coexistence may or may not be possible, depending on the precise form of the trade-off between segregation advantage and negative fitness effects at the individual level. Let 
us now consider the implications of a trade-off for the competition between several distorter alleles. Figure 6 shows the outcome of competition between 11 distorter alleles that all enter the population with the same frequency. The panels of this figure correspond to those of Fig. 5. In the multiplicative model [(a) and (b)] more efficient distorters quickly outcompete less efficient ones, and ultimately a population results where only the most efficient distorter is present. In case of severe individual selection (a) this is not unexpected, since for this scenario already two distorters can coexist only under very special circumstances [Fig. 5(a)]. The outcome in Fig. 6(b) is less obvious, since Fig. 5(b) has shown that under less severe selection coexistence is, at least in principle, possible. That the most efficient distorter $(\sigma=1.0)$ is also dominant in this case is explained by the fact that this distorter cannot be complemented by any of its competitors $\left[w_{i j}=0\right.$, see (15)] and that it is therefore

(a)

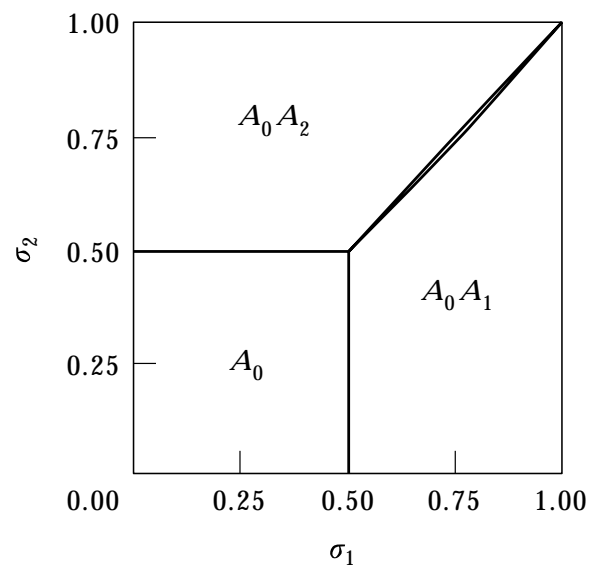

(c)

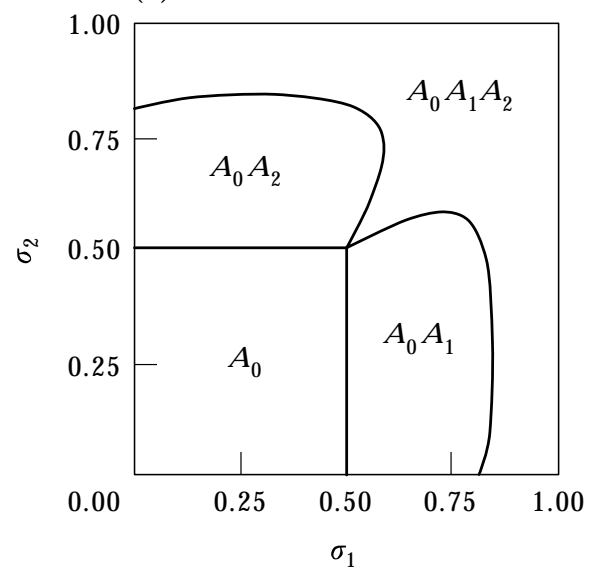

immune against invasion. If this extreme distorter is excluded from consideration, and the range of segregation ratios is limited to, say 0.0 to 0.90 , no distorter allele is competively dominant. However, only a rather moderate degree of polymorphism results, and the allele with the highest segregation ratio reaches by far the highest frequency (results not shown).

In case of an epistatic trade-off [Figs 6(c) and (d)], the outcome of competition is fundamentally different. Here, populations result with a high degree of polymorphism. In (c) the most efficient distorter still reaches the highest frequency, but a number of complementing distorters (with $\sigma_{\mathrm{i}}<\frac{1}{2}$ ) may also persist with appreciable frequencies. In (d), the frequencies of all distorters are more equal, and the segregation ratio is only of marginal importance for the evolutionary success of any specific distorter allele. In fact, distorters with segregation ratios of (b)

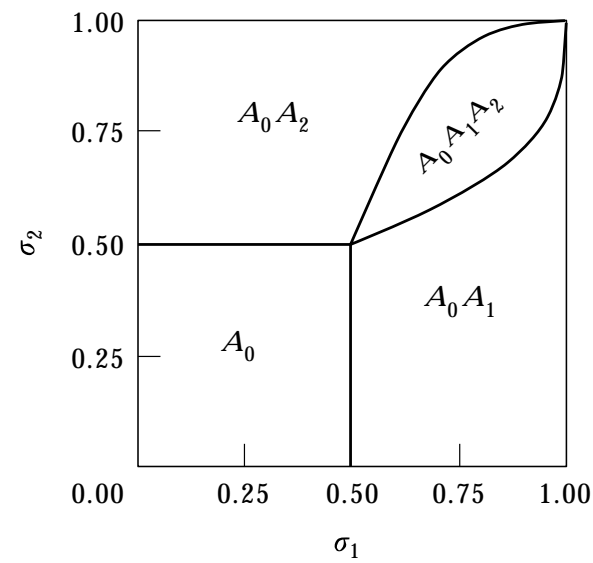

(d)

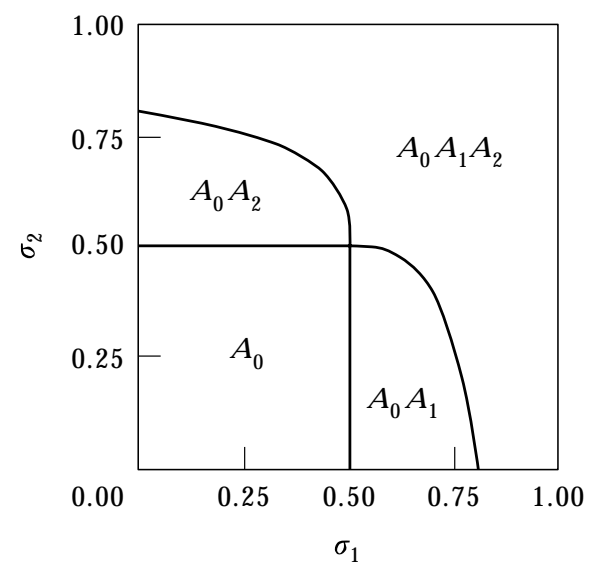

FIG. 5. Outcome of competition between distorters when there is a trade-off between the segregation ratios $\sigma_{i}$ and $\sigma_{j}$, and fitness $w_{i j}=w_{i j}\left(\sigma_{1}, \sigma_{2}\right)$. (a) and (b): multiplicative fitness model (13), and (c) and (d): epistatic fitness model (14). In (a) and (c) $k=\frac{1}{2}$, i.e. a small increase in the segregation ratio leads to a considerable reduction in fitness. In (b) and (d) $k=2$, i.e. a small increase in the segregation ratio reduces fitness to a lesser extent. 
(a)

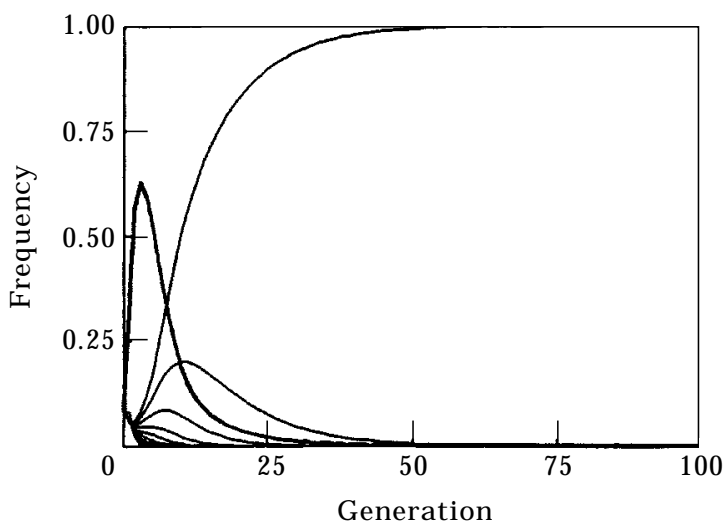

(c)

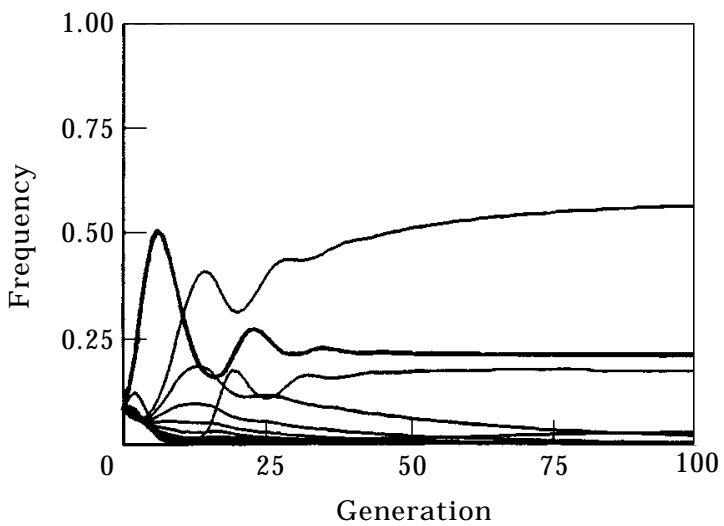

(b)

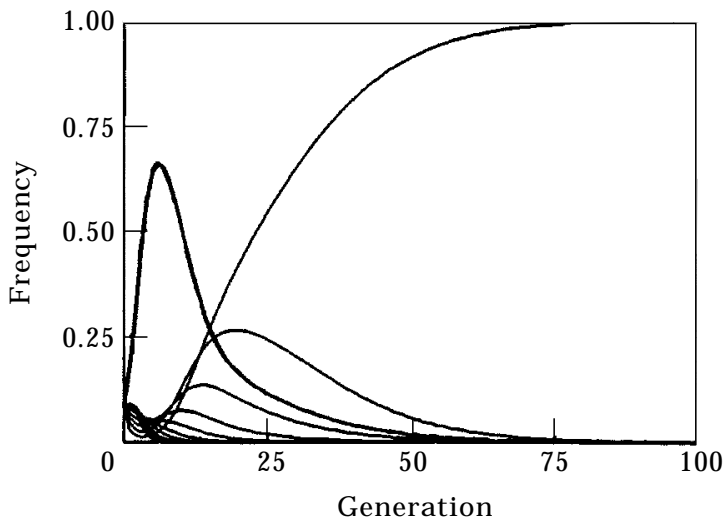

(d)

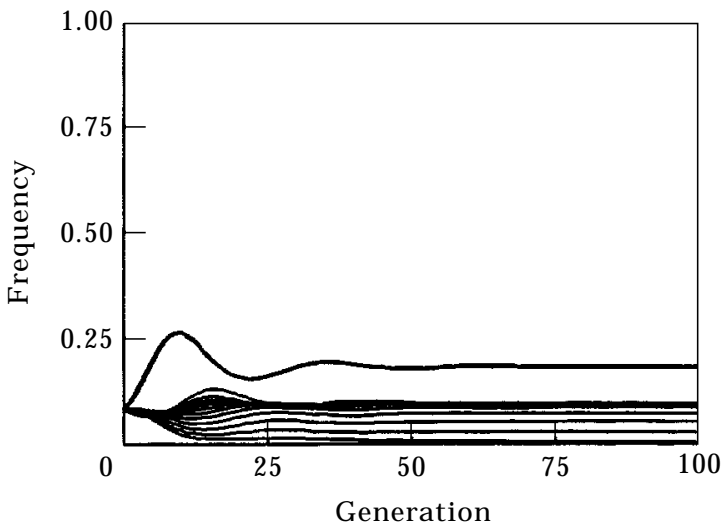

FIG. 6. Outcome of competition between the wildtype (bold line), and a number of distorter alleles $(n=11)$ when there is a trade-off between distorting ability, and fitness in combination with other distorters. The segregation ratio is increased with a stepsize of 0.1 (cf. Fig. 2). The panels correspond to those of Fig. 5. At $t=0$ all alleles are present in equal frequencies.

$0.50-0.70$ reach the highest frequencies. Panel (d) resembles a self-incompatibility system, where every new rare allele is favoured by selection (e.g., Uyenoyama, 1993).

One might question the robustness of the results of Fig. 6 since, as we have seen in Fig. 4, it is not at all obvious that the outcome of competition is independent of the initial conditions. However, simulations show that the complications of Fig. 4 do not arise for the scenarios of Fig. 6 (results not shown): the outcome of competition is always the same, regardless of the order in which the alleles are introduced.

\section{Discussion}

Most studies for the evolution of segregation distortion consider a distorter locus with a wildtype allele and a single distorter allele, and investigate under what conditions modifiers of the segregation ratio will spread (e.g., Prout et al., 1973; Liberman, 1976; Eshel, 1985; Lessard, 1985). In this paper, we take a different approach. We restrict attention to the distorter locus and consider the competition between a large variety of distorter alleles. Hence, we focus on the struggle among distorters, and not on the response of the rest of the genome to segregation distortion.

A crucial assumption of our model is that different distorter alleles may complement another, i.e. that the fitness of individuals heterozygous for two different distorters is higher than that of individuals homozygous for a distorter. In the absence of complementation, only the most efficient distorter will persist in the long run. In contrast, complementation often leads to a systematic advantage of rare distorter alleles, resulting in a high degree of polymorphism. In particular, weak segregation distorters with a low segregation ratio may stably coexist with strong distorters. We showed that if the amount of complementation is fixed, all distorters with a segregation ratio above a certain threshold value will persist, resulting in a population where segregation distortion is virtually absent. If the amount of complementation varies, the outcome of competition 
may be contingent on the initial conditions. If complementation is causally related to distortion, the outcome of competition depends crucially on the details of the trade-off between distortion efficiency and complementing ability.

The occurrence of complementation and a trade-off between distortion efficiency and individual fitness are well-documented (e.g., Temin et al., 1991; Lyon, 1986, 1991). However, other aspects of our simple model are not particularly realistic when confronted with real-world segregation distortion systems such as the $S d$ complex of Drosophila melanogaster, or the $t$ complex of the house mouse. For instance, our model predicts very high distorter frequencies (typically over 0.80 ), often resulting in a low population fitness. The problem of an unrealistically high distorter frequency is shared by many deterministic models of segregation distortion [but see Petras (1967) and Lewontin (1968)]. For the $S d$ complex the discrepancy between expected and observed distorter frequencies is usually explained by taking fitness disadvantages in heterozygous condition (e.g., Temin et al., 1991) or the genetical structure of $S d$ into account (Charlesworth \& Hartl, 1978; Wu \& Hammer, 1991). For the $t$ complex, factors such as inbreeding (Petras, 1967), fitness effects in heterozygous condition (e.g., Johnston \& Brown, 1969), reproductive compensation or kin selection (Charlesworth, 1994), selection at the level of the mating pairs (e.g., Lenington \& Heisler, 1991), or group selection (e.g., Lewontin, 1962; Nunney \& Baker, 1993; van Boven \& Weissing, 1998 b) are usually invoked. It remains to be seen how the outcome of evolution is affected under more realistic scenarios which take these factors into account (van Boven \& Weissing, 1998a).

Our study sheds some light on the current controversy on the relevance of evolutionary stability in the context of long-term evolution (e.g., Weissing, 1996; Marrow et al., 1996). In essence, the ESS approach towards adaptive evolution considers evolution as a series of gene substitution events. According to this view, a monomorphic wildtype population is repeatedly challenged by single mutants. Implicitly, it is assumed that mutants with a fitness disadvantage will be wiped out of the population, while mutants that confer a fitness advantage will invade and spread to fixation. If this view were correct, one would expect that the population should either continue to switch between alternative states (e.g., Liberman, 1991; Stadler, 1996; Godelle \& Reboud, 1997), or converge to a monomorphic ESS population. For instance, Godelle \& Reboud (1997) arrived at this conclusion when they applied the gene substitution approach in the context of segregation distortion. However, these authors a priori excluded the phenomenon of complementation and focused on pairwise interactions between distorter alleles where one of the contestants is always superior over the other. Our study shows the limitations of this approach. In fact, our analysis indicates that rare alleles are systematically favoured, while common alleles are always prone to exploitation. As a result, we expect a high degree of polymorphism instead of the competitive superiority of one distorter allele over all others. Our results furthermore suggest that the complementing ability of a distorter allele may be at least as important as the segregation ratio in determining the outcome of evolution. In contrast to the approach taken by Godelle and Reboud, our model does not permit the integration of both intraand inter-individual fitness into a single measure that determines the outcome of evolution. In our opinion, this may well be the rule in systems where selective forces are acting at different levels.

Catalijne van Oss is gratefully acknowledged for helpful comments on the manuscript. The research of Michiel van Boven is supported by the Life Sciences Foundation (SLW), which is subsidized by the Netherlands Organization for Scientific Research (NWO).

\section{REFERENCES}

BRUCK, D. (1957). Male segregation ratio advantage as a factor in maintaining lethal alleles in wild populations of house mice. Proc. Natl Acad. Sci. U.S.A. 43, 152-158.

Charlesworth, B. (1994). The evolution of lethals in the $t$-haplotype system of the mouse. Proc. Roy. Soc. Lond. Series B. 258, 101-107.

Charlesworth, B. \& Harte, D. L. (1978). Population dynamics of the Segregation Distorter polymorphism of Drosophila melanogaster. Genetics 89, 171-192.

EsHeL, I. (1985). Evolutionary genetic stability of Mendelian segregation and the role of free recombination in the chromosomal system. Am. Natur. 125, 412-420.

Feldman, M. W. \& Otтo, S. P. (1991). A comparative approach to the population-genetics theory of segregation distortion. Am. Natur. 137, 443-456.

Godelle, B. \& Reboud, X. (1997). The evolutionary dynamics of selfish replicators: a two-level selection model. J. theor. Biol. 185, 401-413.

Hartl, D. L. (1970a). Analysis of a general population genetic model of meiotic drive. Evolution 24, 538-545.

Hartl, D. L. (1970b). Population consequences of non-Mendelian segregation among multiple alleles. Evolution 24, 415-423.

Hofbauer, J. \& Sigmund, K. (1988). The Theory of Evolution and Dynamical Systems. Cambridge: Cambridge University Press.

Hurst, L. D. (1993). A model for the mechanism of transmission ratio distortion and for $t$-associated hybrid sterility. Proc. Roy. Soc. Lond. Series B. 253, 83-91.

Johnston, P. G. \& Brown, G. H. (1969). A comparison of the relative fitnesses of genotypes segregating for the $t$ allele in laboratory stocks and its possible effect on gene frequency in mouse populations. Am. Natur. 103, 5-21.

Lenington, S. \& Heisler, I. L. (1991). Behavioral reduction in the transmission of deleterious $t$ haplotypes by wild house mice. Am. Natur. 137, 366-378. 
LESSARD, S. (1985). The role of recombination in the modified theory of autosomal segregation distortion. Theor. Popul. Biol. 28, 133-149.

LEWONTIN, R. C. (1962). Interdeme selection controlling a polymorphism in the house mouse. Am. Natur. 96, 65-78.

Lewontin, R. C. (1968). The effect of differential viability on the population dynamics of $t$-alleles in the house mouse. Evolution 22, 262-273.

Lewontin, R. C. \& Dunn, L. C. (1960). The evolutionary dynamics of a polymorphism in the house mouse. Genetics $\mathbf{4 5}$, 705-722.

Liberman, U. (1976). Modifier theory of meiotic drive: is Mendelian segregation stable? Theor. Popul. Biol. 10, 127-132.

LIBERMAN, U. (1991). On the relation between the instability of ESS in discrete dynamics and segregation distortion. J. theor. Biol. 150, 421-436.

Lyon, M. F. (1986). Male sterility of the mouse $t$-complex is due to homozygosity of the distorter genes. Cell 44, 357-363.

Lyon, M. F. (1991). The genetic basis of transmission-ratio distortion and male sterility due to the $t$-complex. Am. Natur. 137, 349-358

Marrow, P., Johnstone, R. A. \& Hurst, L. D. (1996). Riding the evolutionary streetcar: where population genetics and game theory meet. Trends in Ecol. Evol. 11, 445-446.

Nagylaki, T. (1992). Introduction to Theoretical Population Genetics. New York: Springer-Verlag.

Nunney, L. \& Baker, A. E. M. (1993). The role of deme size, reproductive patterns, and dispersal in the dynamics of $t$-lethal haplotypes. Evolution 47, 1342-1359.

Petras, M. L. (1967). Studies of natural populations of Mus. II. Polymorphism at the $T$-locus. Evolution 21, 466-478.

Prout, T., Bundgandd, J. \& Bryant, S. (1973). Population genetics of modifiers of meiotic drive. I. The solution of a special case and some general implications. Theor. Popul. Biol. 4, 446-465.

Silver, L. M. (1985). Mouse $t$-haplotypes. Ann. Rev. Genet. 19, 179-208.

Silver, L. M. (1993). The peculiar journey of a selfish chromosome: mouse $t$ haplotypes and meiotic drive. Trends Genet. 9, 250-254.

STADLER, B. M. R. (1996). Heteroclinic cycles and segregation distortion. J. theor. Biol. 183, 363-379.

Temin, R. G., Ganetzky, B., Powers, P. A., Lyttle, T. W. Pimpinelli, S., Dimitri, P., Wu, C. I. \& Hiraizumi, Y. (1991). Segregation distortion in Drosophila melanogaster. Genetic and molecular analyses. Am. Natur. 137, 287-331.

Uyenoyama, M. K. (1993). Genetic incompatibility as a eugenic mechanism in: The Natural History of Inbreeding and Outbreeding, (Thornhill, N. W., ed.), pp. 60-73. Chicago: The Chicago University Press.

VAn Boven, M. \& Weissing, F. J. (1998a). Evolution at the mouse $t$ complex: why is the $t$ haplotype preserved as an integral unit? Evolution (submitted).

van Boven, M. \& Weissing, F. J. (1998b). Segregation distortion in a deme-structured population: Opposing demands of gene, individual, and group selection. Journal of Evolutionary Biology (in press)

van Boven, M., Weissing, F. J., Heg, D. \& Huisman, J. (1996). Competition between segregation distorters: Coexistence of 'superior' and 'inferior' haplotypes at the $t$-complex. Evolution 50, 2488-2498.

Weissing, F. J. (1991). Evolutionary stability and dynamic stability in a class of evolutionary normal form games, In: Game Equilibrium Models. I. Evolution and Game Dynamics. (Selten, R. ed.), pp. 29-97. Berlin: Springer-Verlag.

Weissing, F. J. (1996). Genetic versus phenotypic models of selection: can genetics be neglected in a long-term perspective? $J$. Math. Biol. 34, 533-555.

Weissing, F. J. \& VAN Boven, M. (1998). Competition between segregation distorters in monoecious and dioecious populations. Theoretical Population Biology (submitted).

Wu, C. I. \& HAMmeR, M. F. (1991). Molecular evolution of ultraselfish genes of meiotic drive systems, In: Evolution at the
Molecular Level, (Selander, R. K. \& Clark, A. G., eds), pp. 177-203. Sunderland: Sinauer Associates.

\section{APPENDIX}

Consider an allele frequency equilibrium $\mathbf{p}^{*}$ where the wildtype allele $A_{0}$ and $n$ distorter alleles are present with positive frequencies $\left(p_{i}^{*}>0\right.$ for $i=0$, $\ldots, n)$. At equilibrium, all resident alleles have the same marginal fitness: $w_{i}\left(\mathbf{p}^{*}\right)=w_{j}\left(\mathbf{p}^{*}\right)$ for all $i, j \in\{0$, $\ldots, n\}$. If the fitness matrix $\mathbf{W}$ is given by (6), the marginal fitnesses can be written as

$$
w_{0}\left(\mathbf{p}^{*}\right)=1-\frac{1}{2} p_{0}^{*}-\sum_{k=1}^{n} p_{k}^{*} \sigma_{k}
$$

and

$$
w_{i}\left(\mathbf{p}^{*}\right)=p_{0}^{*} \sigma_{i}+\alpha\left(1-p_{0}^{*}-p_{i}^{*}\right),
$$

where $i=1, \ldots, n$. For $i, j \in\{1, \ldots, n\}$, the identity $w_{i}\left(\mathbf{p}^{*}\right)=w_{j}\left(\mathbf{p}^{*}\right)$ implies

$$
w_{i}\left(\mathbf{p}^{*}\right)-w_{j}\left(\mathbf{p}^{*}\right)=\alpha\left(p_{j}^{*}-p_{i}^{*}\right)+\left(\sigma_{i}-\sigma_{j}\right) p_{0}^{*}=0,
$$

or, equivalently

$$
p_{i}^{*}-p_{j}^{*}=\left(\sigma_{i}-\sigma_{j}\right) \frac{p_{0}^{*}}{\alpha} .
$$

Let us now express the equilibrium frequencies in terms of the unweighted mean and variance of the segregation ratios $\sigma_{1}, \ldots, \sigma_{n}$ :

$$
\begin{gathered}
\bar{\sigma}=\frac{1}{n} \sum_{k=1}^{n} \sigma_{k} \\
\operatorname{Var}(\sigma)=\frac{1}{n} \sum_{k=1}^{n}\left(\sigma_{k}-\bar{\sigma}\right)^{2}=\frac{1}{n} \sum_{k=1}^{n} \sigma_{k}^{2}-\bar{\sigma}^{2} .
\end{gathered}
$$

To this end, rewrite (A.3) as

$$
p_{i}^{*}=p_{j}^{*}+\left(\sigma_{i}-\sigma_{j}\right) \frac{p_{0}^{*}}{\alpha},
$$

and take the (unweighted) average of the r.h.s. over all $j=1, \ldots, n$ to obtain

$$
\begin{aligned}
p_{i}^{*}=\frac{1}{n} \sum_{j=1}^{n} p_{j}^{*}+ & \left(\sigma_{i}-\bar{\sigma}\right) \frac{p_{0}^{*}}{\alpha} \\
& =\frac{1}{n}\left(1-p_{0}^{*}\right)+\left(\sigma_{i}-\bar{\sigma}\right) \frac{p_{0}^{*}}{\alpha} .
\end{aligned}
$$


Multiplying both sides with $\sigma_{i}$ and summing over $i=1, \ldots, n$ yields

$$
\sum_{i=1}^{n} p_{i}^{*} \sigma_{i}=\left(1-p_{0}^{*}\right) \bar{\sigma}+\frac{p_{0}^{*}}{\alpha}\left(\sum_{i=1}^{n} \sigma_{i}^{2}-\left(\sum_{i=1}^{n} \sigma_{i}\right) \bar{\sigma}\right),
$$

or

$$
\sum_{i=1}^{n} p_{i}^{*} \sigma_{i}=\left(1-p_{0}^{*}\right) \bar{\sigma}+\frac{n p_{0}^{*}}{\alpha} \operatorname{Var}(\sigma) .
$$

Inserting (A.5) into (A.1), equating (A.1) and (A.2), and taking the sum over $i=1, \ldots, n$ gives the equilibrium frequency of the wildtype allele

$$
p_{0}^{*}=\frac{1-\bar{\sigma}-\alpha\left(1-\frac{1}{n}\right)}{\frac{1}{2}+\frac{n}{\alpha} \operatorname{Var}(\sigma)-\alpha\left(1-\frac{1}{n}\right)},
$$

and, upon insertion into (A.4) also the equilibrium frequency of the distorter alleles.

Consider now a rare allele $A_{n+1}$ that is not present at equilibrium $\left(p_{n+1}^{*}=0\right)$. This allele will spread in the population if its marginal fitness

$$
w_{n+1}\left(\mathbf{p}^{*}\right)=\sigma_{n+1} p_{0}^{*}+\alpha\left(1-p_{0}^{*}\right)
$$

is larger than $w_{1}\left(\mathbf{p}^{*}\right)=\ldots=w_{n}\left(\mathbf{p}^{*}\right)$, the marginal fitness of the resident distorter alleles. Taking the unweighted mean of the r.h.s. of eqn (A.2), we can express the marginal fitness of the resident alleles as

$$
w_{i}\left(\mathbf{p}^{*}\right)=\bar{\sigma} p_{0}^{*}+\alpha\left(1-\frac{1}{n}\right)\left(1-p_{0}^{*}\right)
$$

Hence, the invasion criterion $w_{n+1}\left(\mathbf{p}^{*}\right)>w_{i}\left(\mathbf{p}^{*}\right)$ becomes

$$
\sigma_{n+1}>\bar{\sigma}-\frac{\alpha}{n} \frac{1-p_{0}^{*}}{p_{0}^{*}} .
$$

Insertion of (A.6) into (A.9) leads to criterion (8) for invasion of a rare distorter allele $A_{n+1}$ :

$$
\sigma_{n+1}>\bar{\sigma}-\frac{\frac{\alpha}{n}\left(\bar{\sigma}-\frac{1}{2}\right)+\operatorname{Var}(\sigma)}{1-\bar{\sigma}-\alpha\left(1-\frac{1}{n}\right)} .
$$

Relation (A.10) can be used to derive the minimal segregation ratio $\sigma_{\min }(\alpha)$ needed for successful invasion into a highly polymorphic population. Assume that all distorter alleles with a segregation ratio higher than $\sigma_{\min }(\alpha)$ can persist, while those with a lower segregation ratio cannot. If $n$ is large, $\sigma_{\min }(\alpha)$ is given by

$$
\sigma_{\min }(\alpha)=\bar{\sigma}-\frac{\operatorname{Var}(\sigma)}{1-\bar{\sigma}-\alpha} .
$$

The unweighted mean and variance $\bar{\sigma}$ and $\operatorname{Var}(\sigma)$ of the resident alleles correspond to the mean and variance of the uniform distribution over the interval $\left[\sigma_{\min }(\alpha), 1\right]$ :

$$
\bar{\sigma}=\frac{1+\sigma_{\min }(\alpha)}{2}, \operatorname{Var}(\sigma)=\frac{1}{12}\left[1-\sigma_{\min }(\alpha)\right]^{2} .
$$

Insertion of (A.12) into (A.11) yields (9):

$$
\sigma_{\min }(\alpha)=\left\{\begin{array}{cc}
1-3 \alpha & \text { for } \alpha \leq \frac{1}{3} \\
0 & \text { for } \alpha>\frac{1}{3}
\end{array}\right.
$$

Insertion of (A.13) into (A.12) gives the (unweighted) mean and variance of the segregation ratio,

$$
\begin{gathered}
\bar{\sigma}=\left\{\begin{array}{cl}
1-\frac{3}{2} \alpha & \text { for } \alpha \leq \frac{1}{3} \\
\frac{1}{2} & \text { for } \alpha>\frac{1}{3}
\end{array}\right. \\
\operatorname{Var}(\sigma)=\left\{\begin{array}{cll}
\frac{3}{4} \alpha^{2} & \text { for } & \alpha \leq \frac{1}{3} \\
\frac{1}{12} & \text { for } & \alpha>\frac{1}{3}
\end{array} .\right.
\end{gathered}
$$

The weighted mean of the segregation ratio, $\overline{\mathbf{p}^{*} \sigma}$, is given by (A.5). Since $\lim _{n \rightarrow \infty} p_{0}^{*}=0$, [see eqn (A.6)], the weighted mean segregation ratio is given by

$$
\overline{p^{*} \sigma}=\sum_{k=1}^{n} p_{k}^{*} \sigma_{k}=\bar{\sigma}+\frac{n p_{0}^{*}}{\alpha} \operatorname{Var}(\sigma) .
$$

Insertion of (A.14) into (A.6) shows that the product $n p_{0}^{*}$ is given by

$$
n p_{0}^{*}=\left\{\begin{array}{cc}
\frac{2}{3} & \text { for } \quad \alpha \leq \frac{1}{3} \\
6 \alpha(1-2 \alpha) & \text { for } \quad \alpha>\frac{1}{3}
\end{array}\right.
$$

Finally, inserting (A.14) and (A.16) into (A.15) leads to (10), the weighted mean segregation ratio in a highly polymorphic population

$$
\overline{p^{*} \sigma}=1-\alpha \text {. }
$$

